\title{
Long Period Fiber Grating Based Refractive Index Sensor With Enhanced Sensitivity Using Michelson Interferometric Arrangement
}

\author{
Amit SINGH* \\ Department of Electronics and Communication Engineering, Beant College of Engineering \& Technology, Gurdaspur, \\ Punjab, India \\ *Corresponding author: Amit SINGH \\ E-mail address: er.amitsingh07@gmail.com
}

\begin{abstract}
The long period fiber grating (LPFG) is widely used as a sensor due to its high sensitivity and resolution. However, the broad bandwidth of the attenuation bands formed by the mode coupling between the fundamental core mode and the cladding modes constitutes a difficulty when the device is used as a conventional sensor. To overcome this limitation, a Michelson interferometer-type sensor configuration has been developed, using an LPFG grating pair formed by coating a mirror at the distal end of the LPFG. This sensor configuration is more convenient to use and is able to overcome the limitations of the single LPFG based sensor as the shifts in the attenuation bands being more easily detectable due to the formation of the sharp spectral fringe pattern in the LPFG based Michelson interferometer. In this work, I studied the LPFG based Michelson interferometer as the refractive index sensor and discussed the sensitivity enhancement of the LPFG based Michelson interferometer as a refractive index sensor by employing higher order cladding modes and by reducing the cladding radius. The results demonstrated the $\mathrm{HE}_{17}$ mode with a cladding radius of 62.5 $\mu \mathrm{m}$, in the range of surrounding refractive index (SRI) of $1-1.45$, and its resonant peak showed a wavelength shift of $26.99 \mathrm{~nm} / \mathrm{RIU}$. When the cladding region was further reduced to $24 \mu \mathrm{m}$, the resonant peak showed a wavelength shift of $569.88 \mathrm{~nm} / \mathrm{RIU}$, resulting in a sensitivity enhancement of nearly 21 times. However, as the cladding region was etched further, then the $\mathrm{HE}_{17}$ order cladding mode and higher mode would be cut off. Therefore, the implementation of high sensitivity for SRI sensing with the reduced cladding in the LPFG based Michelson interferometer is dependent on the proper combination of the cladding radius and cladding mode order.
\end{abstract}

Keywords: Long period fiber grating (LPFG), Mach-Zehnder interferometer, self-interfering LPFG (SILPFG), Michelson interferometer, refractive index (RI), reduced cladding, sensitivity

Citation: Amit SINGH, "Long Period Fiber Grating Based Refractive Index Sensor With Enhanced Sensitivity Using Michelson Interferometric Arrangement," Photonic Sensors, 2015, 5(2): 172-179.

\section{Introduction}

The development of various devices for external refractive index sensing has become increasingly important for numerous applications in various industrial processes, biomedical analysis, and environmental monitoring. The widely used fiber optic sensors usually employ the long-period fiber grating (LPFG) [1, 2], etched fiber Bragg grating [3], and plasmon resonance sensing structure [4]. Among these, greater attention has been paid to sensors based on LPFGs because of their high sensitivity, relatively simple fabrication, and simple signal detection. The LPFG promotes the coupling between

Received: 26 September 2014 / Revised version: 9 December 2014

(C) The Author(s) 2015. This article is published with open access at Springerlink.com DOI: $10.1007 / \mathrm{s} 13320-015-0222-7$

Article type: Regular 
the propagating core mode (i.e. the $\mathrm{LP}_{01}$ mode present in the core) and co-propagating cladding modes, $\mathrm{HE}_{1 m}$ (with $m=1,2,3,4, \cdots$ ) in the perturbed region, and the transmission spectrum is formed, which consists of series of attenuation bands at various discrete resonant wavelengths [6].

$$
\lambda_{\text {res }}^{m}=\left(n_{\text {eff,co }}-n_{\text {eff,cl }}^{m}\right) \Lambda
$$

where $\lambda_{\text {res }}$ is the resonant wavelength corresponding to $m$ th cladding mode, $n_{\text {eff,co }}$ is the effective refractive index of the core mode, $n_{\text {eff,cl }}^{m}$ is the effective refractive index of the $m$ th cladding mode, and $A$ is the grating period.

The LPFG is very useful as the refractive index sensor when the refractive index of the surrounding medium changes [5]. The change in the surrounding refractive index (SRI) changes the effective refractive index of the cladding modes and will lead to wavelength shifts in the resonance dips of the LPFG transmission spectrum, which is also governed by (1). These spectral changes are used to measure the surrounding refractive index. The effect of the SRI on the peak resonant wavelength can be expressed by arranging (1) [6]:

$$
\frac{d \lambda_{\mathrm{res}}}{d n_{\mathrm{ext}}}=\frac{d \lambda_{\mathrm{res}}}{d n_{\mathrm{eff}, \mathrm{cl}}^{m}}\left[\frac{d n_{\mathrm{eff,cl}}^{m}}{d n_{\mathrm{ext}}}\right]
$$

where $n_{\text {ext }}$ is the surrounding refractive index. The term in brackets is unique to each coupling condition, and for each cladding mode, the term $\frac{d n_{\text {eff,cl }}^{m}}{d n_{\text {ext }}}$ possesses a different value, hence the LPFG is expected to have a strong dependence on the order of the coupled cladding mode for employing the LPFG as the refractive index sensor [6]. The resolution and sensitivity obtained from single LPFG based sensors can be hampered by large bandwidths belonging to individual attenuation peaks. This effect is remedied by using two in series LPFGs because the resulting spectral fringes have considerably narrower bandwidths [7]. In this technique, the first LPFG couples light from the core mode to various cladding modes. The coupled cladding mode propagates in the cladding and is re-coupled to the core mode by the second LPFG, while the uncoupled core mode continues to propagate through the core region as shown in Fig. 1.

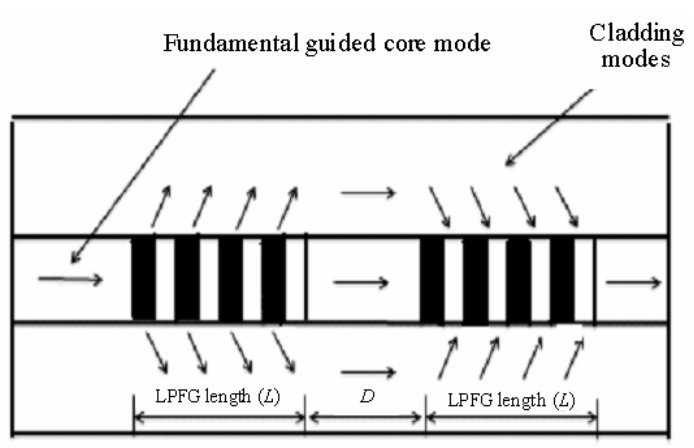

Fig. 1 Schematic representation of the Mach-Zehnder interferometer operation.

The light from these two optical paths interferes through the second LPFG, thereby creating a sharp spectral fringe pattern within the broad loss bands, functioning as an in-fiber Mach-Zehnder interferometer (MZI) [8]. The in-fiber MachZehnder interferometer is used in the transmission mode, where $D$ is the gap separating the two in series LPFGs.

Another interferometer operating in the reflection mode has been demonstrated by formation of a mirror at the end of a fiber containing an LPFG. This simple structure has the same spectral properties as the in series LPFG pairs but offers advantages such as the compact size and the ability to use a single-ended probe in some applications. The shape of this interferometer is analogous to the Michelson interferometer (MI) and also termed as the self-interfering long period fiber grating (SILPFG) [9]. In the SILPFG, the optical power lost from the fundamental core mode as a result of mode coupling within the grating is re-coupled into the core mode when travelling in the opposite direction subsequent to having been reflected by the fiber mirror. Advantages of this sensor configuration include the fact that the fringes in its reflectance spectrum may be used for high-resolution sensing, 
and it does not require the manufacture of two identical gratings, which is a technically challenging task [10].

\section{Operating principle of LPFG based Michelson interferometer}

The operating principle of the LPFG based Michelson interferometer is almost the same as the LPFG based MZIs. The main difference is the existence of a reflective mirror present in the LPFG based Michelson interferometer. Since MIs use reflection modes, they are compact and handy in practical uses and installation [9] as shown in Fig.2.

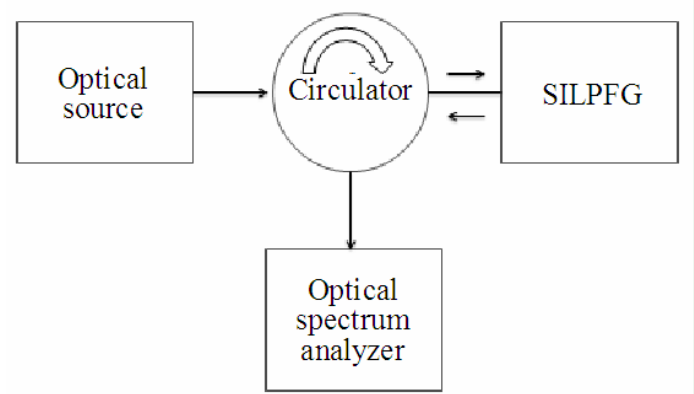

Fig. 2 LPFG based Michelson interferometer sensor (SILPFG).

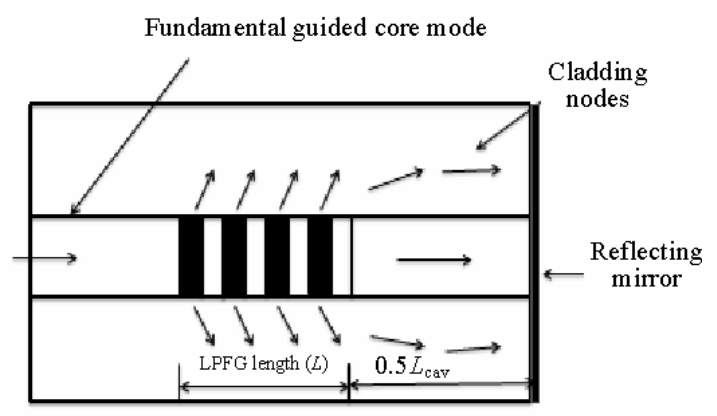

(a)

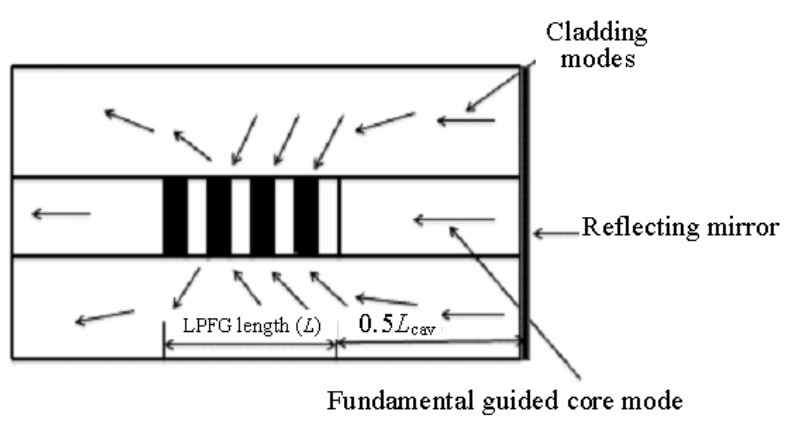

(b)

Fig. 3 Light propagation in the SILPG: (a) light propagation in the SILPFG during the forward propagation path and (b) light propagation in the SILPFG after reflection from the mirror.
As shown in Fig.3(a), light propagating as core mode $\mathrm{LP}_{01}$ encounters the LPFG, and portion of the optical power couples with various cladding modes $\left(\mathrm{LP}_{0 m}\right)$, while the residue remains in the core and propagates as the fundamental core mode, which is similar to the transmission spectrum of the single LPFG shown in Fig. 4. The various parameters used in the simulation procedure are given in Table 1.

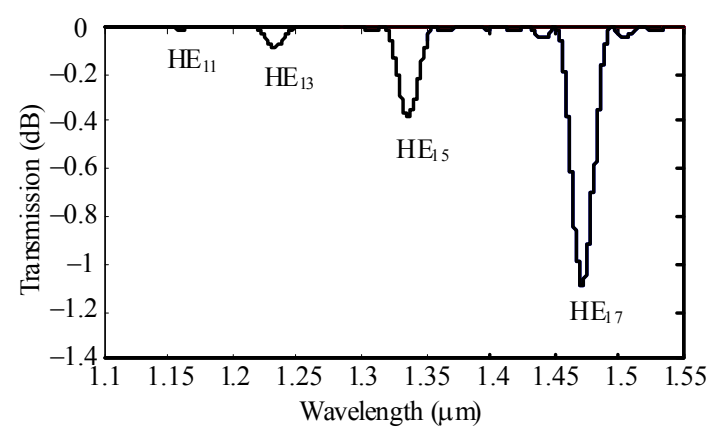

Fig. 4 Transmission spectrum of the LPFG.

Table 1 Parameters used in simulation.

\begin{tabular}{cc}
\hline Parameters & Values used \\
\hline Core radius $\left(a_{\mathrm{co}}\right)$ & $3.65 \mu \mathrm{m}$ \\
Cladding radius $\left(a_{\mathrm{cl}}\right)$ & $62.5 \mu \mathrm{m}$ \\
Core refractive index $\left(n_{\mathrm{co}}\right)$ & 1.465 \\
Cladding refractive index $\left(n_{\mathrm{cl}}\right)$ & 1.46 \\
Grating period $(\Lambda)$ & $455 \mu \mathrm{m}$ \\
Free space wavelength $(\lambda)$ & $1.310 \mu \mathrm{m}$ \\
\hline
\end{tabular}

When the end of the fiber has a reflective mirror across both the core and cladding materials [11] as shown in Fig. 3(b), then the light waves in both the core and cladding are reflected back towards the grating, thereby retracing their paths along the respective regions of the fiber to the LPFG. The optical power present in the core after reflecting from the fiber mirror, is divided once again upon encountering the LPFG: a portion of the light remains guided in the fundamental core mode, whereas the remaining power is coupled with various cladding modes and is eventually lost. Similarly, the optical power that exists in the cladding mode returns to the grating from the fiber reflector, and a proportion of cladding optical power continues to propagate in the cladding, where it also vanishes due to losses, and the remaining optical power is coupled back into the core by the LPFG 
[12]. It is the latter light wave that causes interference with the backward propagating guided mode already present in the core since both of these waves travel away from the grating, and yet they are guided along the core material with different phase characteristics. Therefore, the light wave that is recombined in the core and the light wave that is originally in the core interfere with each other to form the same spectral fringes observed for twin LPFGs, shown in Fig. 5 In this case, I chose the cavity length of $20 \mathrm{~mm}$.

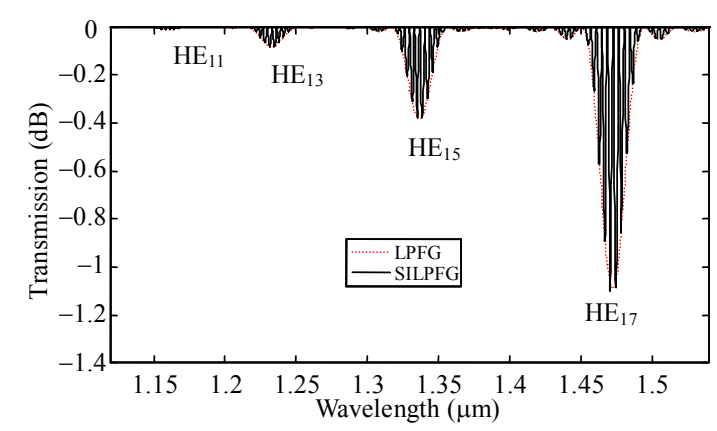

Fig. 5 Comparison between the full reflectance spectrum of Michelson interferometer with a cavity length of $L_{\mathrm{cav}}=200 \mathrm{~mm}$ and transmission spectrum of the individual LPFG.

The main difference between self-interfering LPFGs and an ideal twin LPFG structure is the fact that the cavity length $(D)$ of the LPFG pairs corresponds to twice the distance between the LPFG and the mirrored fiber end in the SILPFG $\left(0.5 L_{\text {cav }}\right)$ [12]. Therefore, the less fiber is necessary in the construction of the SILPFG than that for a twin LPFG, which also requires an additional, nearly identical grating.

The sensitivity and resolution of the SILPFG based sensor can be easily adjusted by changing the length of the fiber cavity between the LPFG and the mirrored end-face, since the interference pattern's period (fringe spacing) depends on the optical path lengths present in the SILPG $[11,12]$. Now I will investigate the variation of the fringe spacing in the SILPFG in terms of the cavity length [13]. When I reduce the cavity length to $100 \mathrm{~mm}$, in this case, the fringe spacing increases as shown in Fig. 6 .
For better clarity, in Fig. 7, the closer view of $\mathrm{HE}_{15}$ attenuation band fringe spacing due to the variation in the cavity length is shown. The special frequency of the interference pattern is directly relational to the cavity length of the interferometer. This enables the designer to increase the phase sensitivity by increasing the cavity length. The sensor with the longer cavity has the higher sensitivity, but resulting in the smaller fringe visibility [11].

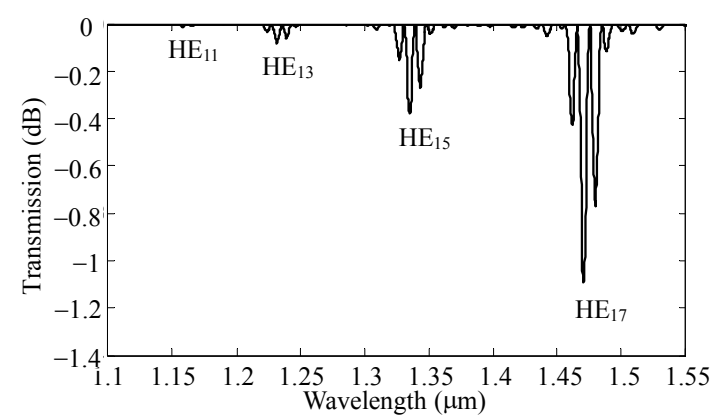

Fig. 6 Theoretical reflectance of a Michelson interferometer with a cavity length of $L_{\mathrm{cav}}=100 \mathrm{~mm}$.

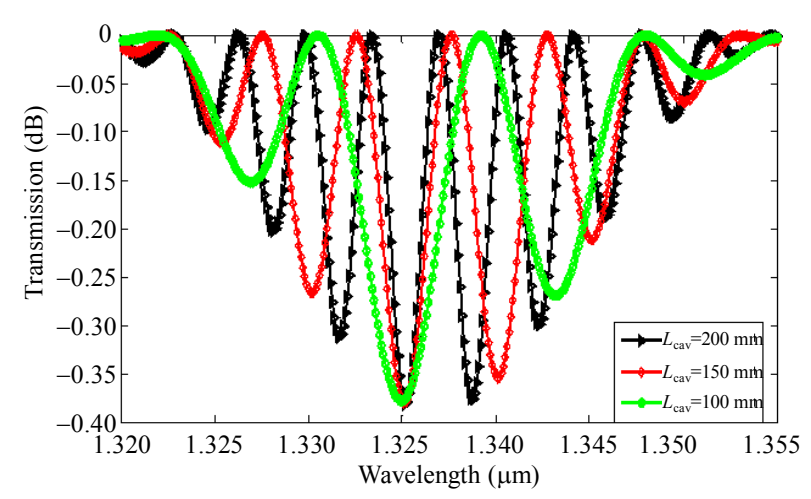

Fig. $7 \mathrm{HE}_{15}$ attenuation band of the Michelson interferometer simulated for different cavity lengths.

\section{SILPFG as refractive index sensor}

For using the SILPFG as a sensor, the path length differences are induced in two arms of the interferometer as a result of external perturbations [11]. These differences in optical path lengths produce corresponding phase shifts in the interferometric fringe pattern, this time in the reflectance of the SILPFG. Figure 8 shows the phase change as a function of the refractive index for the $\mathrm{HE}_{15}$ mode of the SILPFG. 


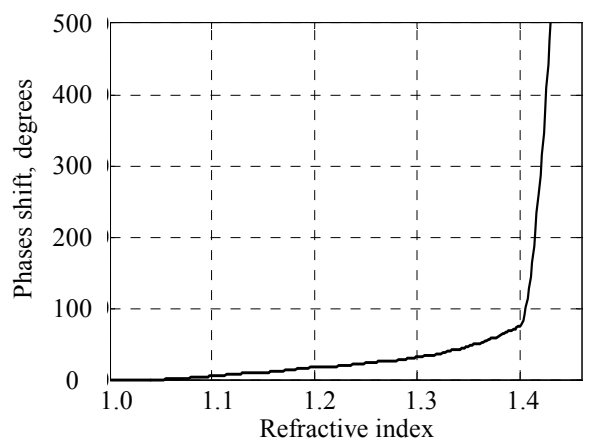

Fig. 8 Measured phase change as a function of the refractive index for $\mathrm{HE}_{15}$ mode of the SILPFG.

Now I will study the behavior of the LPFG spectrum and SILPFG for refractive index sensing of the $\mathrm{HE}_{15}$ mode as shown in Fig. 9. In our discussion, I choose $L_{\mathrm{cav}}=100 \mathrm{~mm}$ for better fringe visibility, and corresponding to this, I have three fringes of the SILPFG spectrum, which are termed as Fringe 1, Fringe 2, and Fringe 3, where Fringe 1 is located at $1.326900 \mu \mathrm{m}$, Fringe 2 is located at $1.334953 \mu \mathrm{m}$, and Fringe 3 is located at $1.343199 \mu \mathrm{m}$.

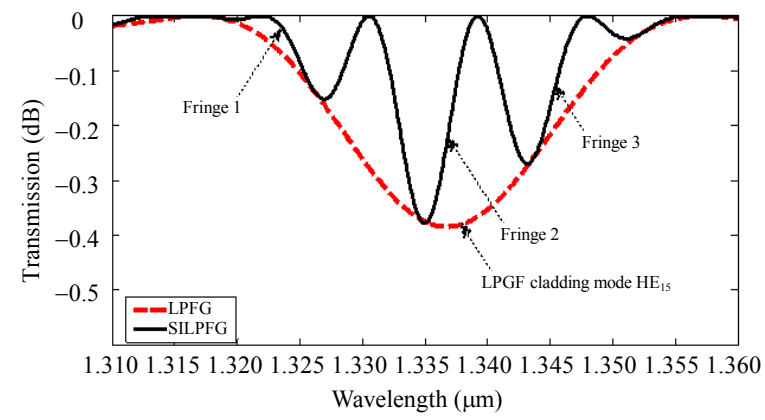

Fig. $9 \mathrm{HE}_{15}$ attenuation band of the LPFG and Michelson interferometer (SILPFG) simulated with cavity lengths of $100 \mathrm{~mm}$.

Figure 10 shows the interference fringes corresponding to the $\mathrm{HE}_{15}$ cladding mode resonance band. As the surrounding refractive index increases, the $\mathrm{HE}_{15}$ resonant band of the LPFG and all three fringes within this resonant band of the SILPFG shift towards shorter wavelengths compared with that of air (i.e. $n_{\mathrm{ext}}=1$ ), and the visibility deteriorates as the surrounding refractive index approaches the cladding refractive index. In the surrounding refractive index range of $1-1.45$, all the three fringes show the same wavelength shift of
$16.80 \mathrm{~nm} / \mathrm{RIU}$. In our latter discussion, the fringe with the deepest attenuation (Fringe 2) is traced as the external RI increases from 1.000 to 1.45 .

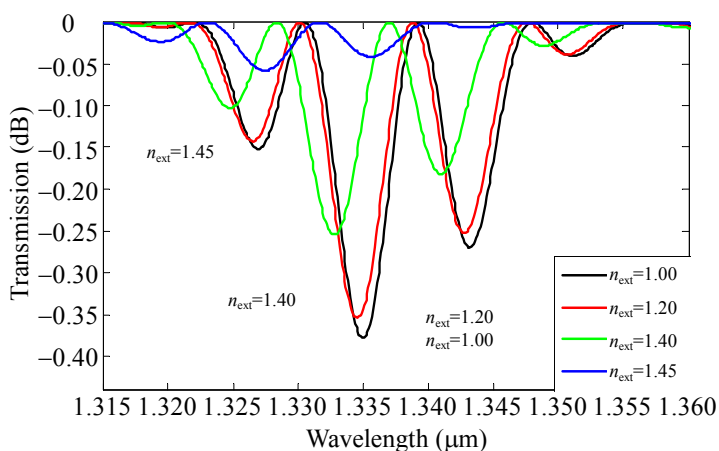

Fig. 10 Reflection spectrum of interference fringes in a $\mathrm{HE}_{15}$ cladding mode and its fringe shift of resonant peaks corresponding to $n_{\text {ext }}=1,1.2,1.4$, and 1.45.

Now I will compare the SILPFG and LPFG as the refractive index sensor for better understanding. Figure 11 shows the dependence of the $\mathrm{HE}_{15}$ mode due to the surrounding refractive index for the long period fiber grating (LPFG). From this figure, as the external refractive index increases, there is a blue shift in resonant peak wavelengths, and the coupling coefficient also decreases. In the refractive index range of $1-1.45$, the resonant peak shows a wavelength shift of $16.80 \mathrm{~nm} / \mathrm{RIU}$.

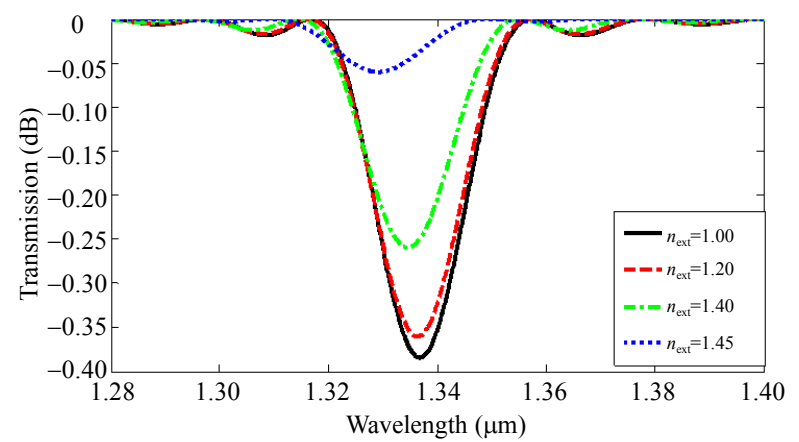

Fig. 11 Spectrum of $\mathrm{HE}_{15}$ in the case of the LPFG (the shift of resonant peaks are at $1.336999 \mu \mathrm{m}, 1.336549 \mu \mathrm{m}$, $1.334747 \mu \mathrm{m}$, and $1.329445 \mu \mathrm{m}$ corresponding to $n_{\mathrm{ext}}=1,1.2$, 1.4 , and 1.45 .

In Fig. 12, the comparison between wavelength shifts of various fringes of the SILPFG and LPFG in the case of the $\mathrm{HE}_{15}$ mode in the refractive index range of $1-1.45$ is shown. In this figure, although the essential sensitivity remains the same in the case of both long period fiber grating based refractive index sensor and self-interfering long period fiber 
grating based refractive index sensor, but the actual sensitivity in the case of the SILPFG based refractive index sensor is much improved due to the narrow bandwidth as seen in Figs. 9 and 10.

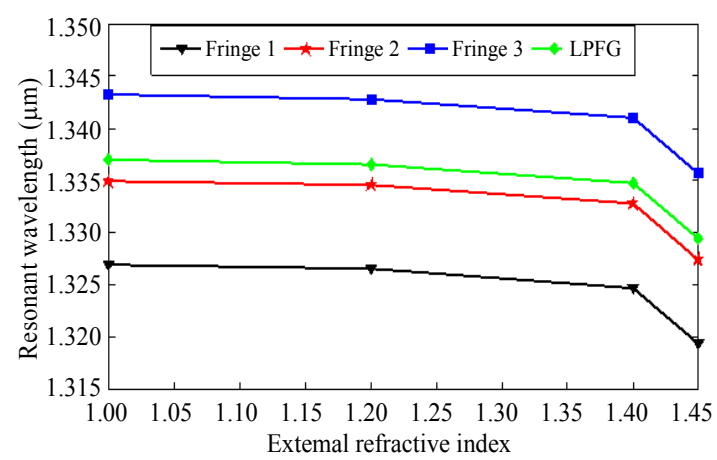

Fig. 12 Comparison between wavelength shifts of various fringes of the SILPFG based refractive index sensor and LPFG based refractive index sensor the for $\mathrm{HE}_{15}$ mode in a external refractive index range of $1-1.45$.

\section{Sensitivity improvement of SILPFG as refractive index sensor}

Now I will study the enhanced sensitivity of the SILPFG based refractive index sensor by using the higher order cladding mode $\mathrm{HE}_{17}$ as shown in Fig. 13. From this figure, we have noticed that when SRI increases from 1 to 1.45 , there is a resonant peak showing a wavelength shift of $26.99 \mathrm{~nm} / \mathrm{RIU}$. Comparision with $\mathrm{HE}_{15}$ and $\mathrm{HE}_{17}$ modes shows the larger variation of the resonant wavelength shift for the same SRI range of $1-1.45$, resulting in a sensitivity enhancement of nearly 1.6 times.

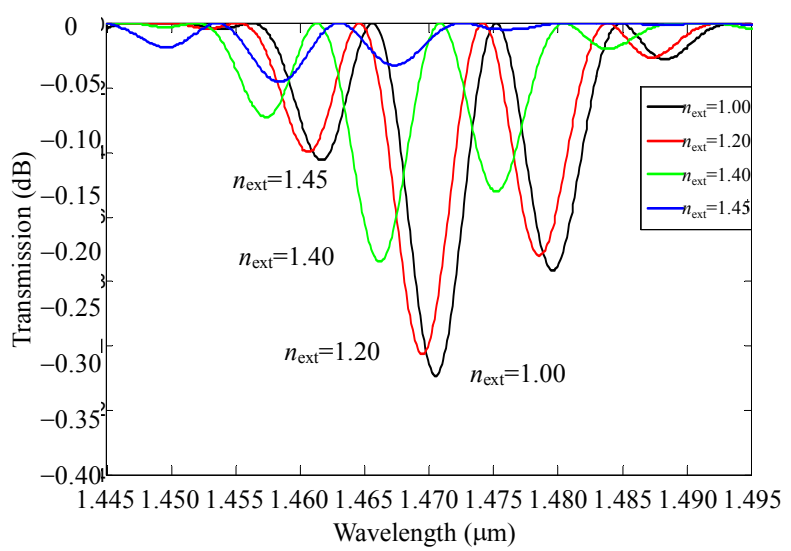

Fig. 13 Reflection spectrum of $\mathrm{HE}_{17}$ based LPFG (the shifts of resonant peaks are at $1.470499 \mu \mathrm{m}, 1.469499 \mu \mathrm{m}$, $1.466145 \mu \mathrm{m}$, and $1.458352 \mu \mathrm{m}$ corresponding to $n_{\mathrm{ext}}=1,1.2$, 1.4 , and 1.45).
Now we will study the effect of the $\mathrm{HE}_{17}$ based SILPFG when the cladding region is etched. Actually when we etch the cladding region, then the number of cladding modes supported will be lesser and lesser. In other words, the reduction in the cladding radius results in a cut-off of the existing highest-order cladding modes [14].

Firstly, I will discuss the variation in the phase shift of the $\mathrm{HE}_{17}$ mode fringe pattern due to different SRIs, when the cladding region is etched as shown in Fig. 14. From this figure, as the cladding region is reduced, then the phase shift increases with an increase in the refractive index.

Now I will study the effect of the $\mathrm{HE}_{17}$ based SILPFG when the cladding radius is etched to $40 \mu \mathrm{m}$ as shown in Fig. 15. From this figure, there is a large wavelength shift as the refractive index increases from 1 to 1.45 . In the SRI range of $1-1.45$, the resonant peaks shows a wavelength shift of $100.64 \mathrm{~nm} / \mathrm{RIU}$.

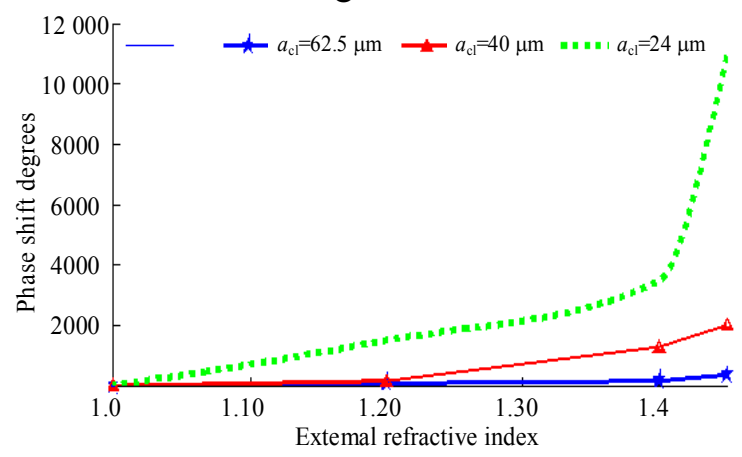

Fig. 14 Measured phase change as a function of the refractive index for the $\mathrm{HE}_{17}$ mode in the SILPFG for different cladding radii.

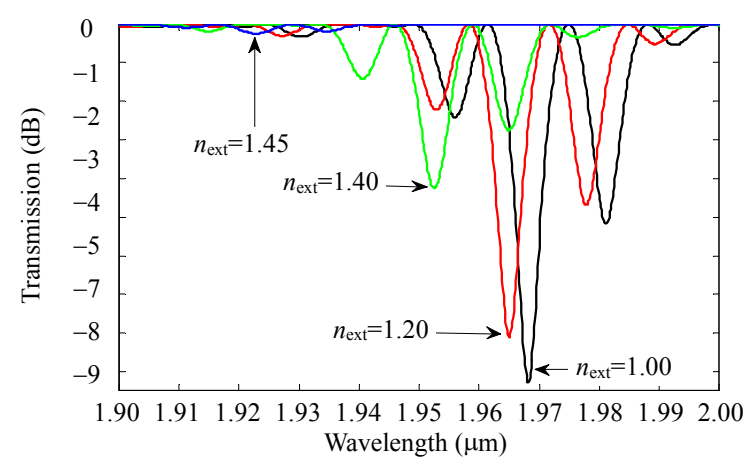

Fig. 15 Reflection spectrum of the $\mathrm{HE}_{17}$ based SILPFG [the shifts of resonant peaks are at $1.968199 \mu \mathrm{m}, 1.965049 \mu \mathrm{m}$, $1.952599 \mu \mathrm{m}$, and $1.922910 \mu \mathrm{m}$ corresponding to $n_{\mathrm{ext}}=1,1.2$, 1.4 , and $\left.1.45\left(a_{\mathrm{cl}}=40 \mu \mathrm{m}\right)\right]$. 
Now I will study the effect of the $\mathrm{HE}_{17}$ based SILPFG when the cladding radius is etched to $24 \mu \mathrm{m}$ as shown in Fig. 16. From this figure, there is much larger wavelength shift as the refractive index increases from 1 to 1.45 . In the SRI range of $1-$ 1.45 , there is a resont peak showing a wavelength shift of $569.88 \mathrm{~nm} / \mathrm{RIU}$.

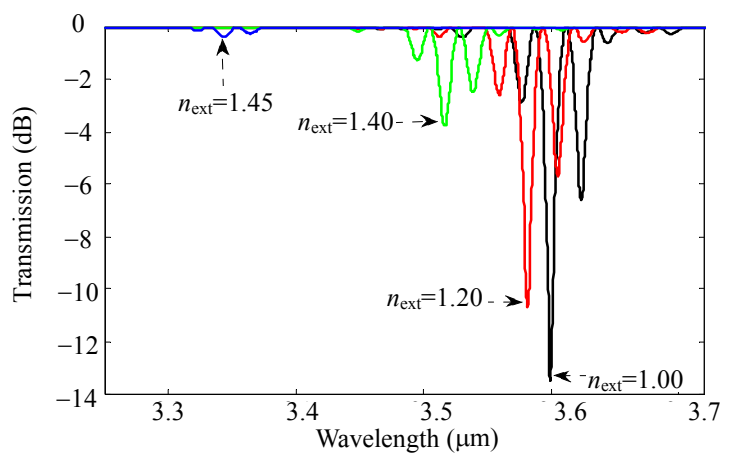

Fig. 16: Reflection spectrum of the $\mathrm{HE}_{17}$ based SILPFG [the shifts of resonant peaks are at $3.599200 \mu \mathrm{m}, 3.581199 \mu \mathrm{m}$, $3.516299 \mu \mathrm{m}, 3.342750 \mu \mathrm{m}$ corresponding to $n_{\mathrm{ext}}=1,1.2,1.4$, and $\left.1.45\left(a_{\mathrm{cl}}=40 \mu \mathrm{m}\right)\right]$.

Therefore, the resonant wavelength shifts and thus the sensitivity are dramatically enhanced as the cladding radius decreases.

In Fig. 17, the resonant wavelength shifts of the $\mathrm{HE}_{17}$ fringe shifts as the function of variation in the SRI with three different values of cladding radii $62.5 \mu \mathrm{m}, 40 \mu \mathrm{m}$, and $24 \mu \mathrm{m}$ are shown.

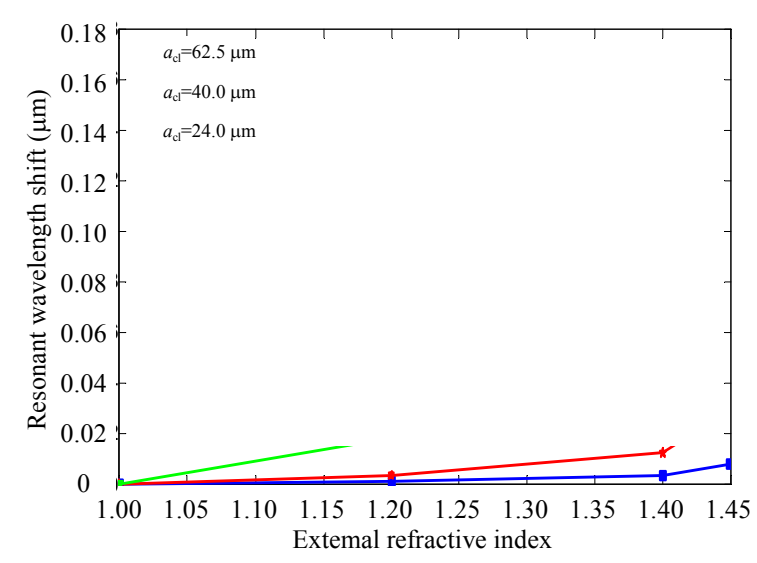

Fig. 17 Resonant wavelength shift of the $\mathrm{HE}_{17}$ mode fringe pattern versus SRI.

In our case, if we require cladding modes up to $\mathrm{HE}_{17}$ to propagate, then the cladding radius will be etched to nearly $24 \mu \mathrm{m}$. When etching the cladding region more, then the $\mathrm{HE}_{17}$ mode will be cut off, and when the cladding region increases, then more cladding modes will propagate, and the sensitivity decreases in the case of the $\mathrm{HE}_{17}$ mode. But in that case, we have to choose the higher order cladding mode such as $\mathrm{HE}_{19}$ for enhancing the sensitivity. Therefore, it is necessary to select a suitable combination of the cladding radius and cladding mode order for high sensitivity with consideration of the varying range of SRI.

\section{Conclusions}

In this paper, an LPFG based Michelson interferometer is presented, which is also termed as the SILPFG that includes a long-period grating operating in the reflection mode for refractive-index sensing. I have further discussed the sensitivity enhancement of the LPFG based Michelson interferometer as the refractive index sensor by employing higher order cladding modes and by reducing the cladding radius. The results show the $\mathrm{HE}_{17}$ mode with the cladding radius of $62.5 \mu \mathrm{m}$, in the range of SRI of $1-1.45$, and the resonant peak shows a wavelength shift of $26.99 \mathrm{~nm} / \mathrm{RIU}$. When the cladding region is further reduced to $24 \mu \mathrm{m}$, the resonant peak shows a wavelength shift of $569.88 \mathrm{~nm} / \mathrm{RIU}$, resulting in the sensitivity enhancement of nearly 21 times. This device offers the advantages of the small size, ease of use, high sensitivity, and potential for various applications in the future use.

Open Access This article is distributed under the terms of the Creative Commons Attribution License which permits any use, distribution, and reproduction in any medium, provided the original author(s) and source are credited.

\section{References}

[1] A. M. Vengsarkar, P. J. Lemaire, J. B. Judkins, V. Bhatia, T. Erdogan, and J. E. Sipe, "Long-period fiber gratings as band-rejection filters," Journal of Lightwave Technology, 1996, 14(1): 58-65.

[2] T. Erdogan, "Cladding-mode resonances in short- and long-period fiber grating filters," Journal of the 
Optical Society of America A, 1997, 14(8): 1760-1773.

[3] K. Shroeder, W. Ecke, R. Mueller, R. Willsch, and A. Andreev, "A fibre Bragg grating refractometer," Measurement Science and Technology, 2001, 12(7): 757-764.

[4] R. Slavik, J. Homola, J. Ctyroky, and E. Brynda, "Novel spectral fiber optic sensor based on surface plasmon resonance," Sensors and Actuators B: Chemical, 2001, 74(1-3): 106-111.

[5] Q. Huang, Y. Yu, Z. Ou, X. Chen, J. Wang, P. Yan, et al., "Refractive index and strain sensitivities of a long period fiber grating," Photonic Sensors, 2014, 4(1): 92-96.

[6] V. Bhatia, "Applications of long-period gratings to single and multi-parameter sensing," Optics Express, 1999, 4(11): 457-466.

[7] B. H. Lee and J. Nishii, "Notch filters based on cascaded multiple long-period fibre gratings," Electronics Letters, 1998, 34(19): 1872-1873.

[8] O. Duhem, J. F. Henninot, and M. Douay, "Study of in fiber Mach-Zehnder interferometer based on two spaced 3-dB long period gratings surrounded by a refractive index higher than that of silica," Optics Communications, 2000, 180(4-6):
$255-262$.

[9] L. Alwis, T. Sun, and K. T. V. Grattan, "Fibre optic long period grating-based humidity sensor probe using a Michelson interferometric arrangement," Sensors and Actuators B: Chemical, 2013, 178: 694-699.

[10] B. H. Lee and J. Nishii, "Self-interference of long-period fibre grating and its application as temperature sensor," Electronics Letters, 1998, 34(21): 2059-2060.

[11] P. L. Swart, "Long-period grating Michelson refractometric sensor," Measurement Science and Technology, 2004, 15(8): 1576-1580.

[12] A. V. Brakel, "Sensing characteristics of an optical fibre long-period grating Michelson refractometer," Ph.D. dissertation, Rand Afrikaans University, Johannesburg, 2004.

[13]M. G. Xu, R. Maaskant, M. M. Ohn, and A.T. Alavie, "Independent tuning of cascaded long period fibre gratings for spectral shaping," Electronics Letters, 1997, 33(22): 1893-1894.

[14] H. Chen and Z. Gu, "Characteristics of a long-period fiber grating with reduced cladding for refractive index sensing," Journal of Modern Optics, 2011, 58(18): 1659-1665. 